compared with children who received placebo $(4.1 \pm 2.0$ versus $6.2 \pm 3.0$, respectively; $P<0.01)$. In particular, $L$. reuteri-treated children were less likely than placebo-treated children to report new-onset or worsened abdominal distension, disorders of defecation, epigastric pain, eructation and halitosis.

The authors note that $L$. reuteri improves the overall tolerability of $H$. pylori eradication therapy. They speculate that $L$. reuteri might reduce epigastric pain and eructation by outcompeting $H$. pylori for binding sites on gastric epithelial cells, which might reduce $H$. pylori bacterial load.

Original article Lionetti E et al. (2006) Lactobacillus reuteri therapy to reduce side-effects during anti-Helicobacter pylori treatment in children: a randomized placebo controlled trial. Aliment Pharmacol Ther 24: 1461-1468

\section{Detection of advanced neoplasia during colorectal screening more likely in males}

A new study has suggested that, along with age $>49$ years and family history of colorectal cancer, male sex is an important predictor of the detection of advanced neoplasia during colorectal screening. Regula and colleagues suggest that colonoscopy guidelines should, therefore, be refined to include this parameter.

Regula and colleagues performed a crosssectional analysis of data from 50,148 patients (aged 40-66 years) who participated in a national, colonoscopy-based screening program in Poland. Multivariate logistic regression was used to identify associations between patient characteristics and advanced neoplasia in two-thirds of the cohort, and these associations were confirmed in the remainder. Advanced neoplasia (i.e. cancer or adenoma $\geq 10 \mathrm{~mm}$ in diameter, high-grade dysplasia, villous or tubulovillous histologic characteristics, or any combination thereof) was detected in 2,553 (5.9\%) participants aged 50-66 years and in $243(3.4 \%)$ participants aged 4049 years. Analysis showed that male sex was independently associated with advanced neoplasia (odds ratio 1.73; 95\% Cl 1.52-1.98). This association remained after adjustment for colonoscopic factors, family history and age (odds ratio 1.98; 95\% Cl 1.83-2.14). The number needed to screen in order to detect one advanced neoplasia was markedly lower in men than in women of similar family history and age (for example, in the 40-49-year agegroup, the number needed to screen was 23 in men and 36 in women).

The authors conclude that modification of colonoscopy screening recommendations would maximize the diagnostic yield of screening, and optimize resource use.

Original article Regula J et al. (2006) Colonoscopy in colorectal-cancer screening for detection of advanced neoplasia. N Engl J Med 355: 1863-1872

\section{High risk of recurrence in patients with hepatitis-C-related liver cancer}

Patients with hepatitis-C-related hepatocellular carcinoma (HC-HCC) have a higher risk of recurrence after surgery than those with hepatitis-B-related hepatocellular carcinoma (HB-HCC), according to researchers in Japan. Sasaki et al. conducted a long-term follow-up of patients who underwent surgery for HB-HCC $(n=66)$ or HC-HCC $(n=351)$ between January 1990 and December 1999.

The median follow-up was 11.0 years for patients with $\mathrm{HB}-\mathrm{HCC}$ and 10.1 years for those with HC-HCC. Recurrence occurred in 39 (59\%) patients with HB-HCC and $282(80 \%)$ with $\mathrm{HC}-\mathrm{HCC}$. The odds ratio for risk of recurrence for HC-HCC compared with HB-HCC was 1.93. The 3-year, 5-year and 10-year disease-free survival rates were $57 \%, 54 \%$ and $28 \%$, respectively in the HB-HCC group and $40 \%, 24 \%$ and $12 \%$, respectively in the HC-HCC group.

Although the recurrence rates were similar for the two viral groups during the first 2 years of follow-up, the long-term prognosis was considerably worse in patients with $\mathrm{HC}-\mathrm{HCC}$, probably because of a higher frequency of metachronous carcinogenesis in these patients. The authors believe that HCC recurrence in both groups was probably caused by intrahepatic metastasis during the first 2 years. They conclude that the type of viral infection must be taken into account when devising treatment strategies for hepatitis-infected patients with HCC.

Original article Sasaki Y et al. (2006) Risk of recurrence in a long-term follow-up after surgery in 417 patients with hepatitis B- or hepatitis C-related hepatocellular carcinoma. Ann Surg 244: 771-780 